\title{
Illumination of exciton migration in rodlike luminescent conjugated polymers by single-molecule spectroscopy
}

\author{
C. F. Wang, ${ }^{1,3}$ J. D. White, ${ }^{1,2}$ T. L. Lim, ${ }^{1,2}$ J. H. Hsu, ${ }^{1}$ S. C. Yang, ${ }^{1,4}$ W. S. Fann, ${ }^{1,3}$ K. Y. Peng, ${ }^{5}$ and S. A. Chen ${ }^{5}$ \\ ${ }^{1}$ Institute of Atomic and Molecular Sciences, Academia Sinica, P.O. Box 23-266, Taipei, Taiwan, Republic of China \\ ${ }^{2}$ Centre for High Speed and Broadband Networking, Multimedia University, Malaysia \\ ${ }^{3}$ Department of Physics, National Taiwan University, Taipei, Taiwan, Republic of China \\ ${ }^{4}$ Department of Physics, Tamkang University, Taipei, Taiwan, Republic of China \\ ${ }^{5}$ Department of Chemical Engineering, National Tsing-Hwa University, Hsinchu, Taiwan, Republic of China
}

(Received 11 July 2002; published 13 January 2003)

\begin{abstract}
Single-molecule spectroscopy is used to study the time-dependent spectral behavior of a short rodlike Poly(phenylene vinylene) (PPV) derivative polymer spin cast in a polystyrene matrix. The fluorescent time trace is characterized by stepwise intensity emission with constant spectral composition, punctuated by abrupt intensity changes, which are usually accompanied by abrupt spectral changes. In contrast to coiled long chain polymers, defect-free rodlike polymers exhibit multiple-emission sites, each with its characteristic invariant spectrum. The distribution of spectral jumps in the emission spectrum reflects the distribution of the effective conjugation length. This implies the energy transfer (i.e., thermalized exciton migration) along the polymer backbone is inefficient. A static disorder induced conjugation length distribution model with limited energy transfer can be used in understanding the photophysics of an isolated polymer.
\end{abstract}

DOI: 10.1103/PhysRevB.67.035202

\section{INTRODUCTION}

The nature of exciton migration along a polymer chain is a fundamental problem in luminescent conjugated polymer physics. Questions close to the heart of this problem are: "Does a thermalized exciton migrate to the lower energy sites before emitting a photon?" and "How does the migration depends on the conformation and structure?" In investigating the femtosecond luminescence dynamics of Poly(phenylene vinylene) (PPV) films, Kersting and co-workers observed a transient redshift of the spectra, which they attributed to ultrafast energy relaxation of the optical excitations. ${ }^{1}$ In studies of the oxidization of PPV films, Rothberg and co-workers indicate that exciton diffusion in the polymer thin films can be 400 repeat units. ${ }^{2}$ Work in singlemolecule spectroscopy is shedding light on this topic. Yu et $a l^{3}$ observed a spectral blueshift when a polymer goes from "fully on" level to "intermediate intensity" level in MEH-PPV. They attributed this to rapid directional energy transfer. Huser et al. ${ }^{4}$ observed in tightly folded chains of MEH-PPV excitation energy being efficiently transferred to the lowest energy excitons. This they attributed to strong intrachain interaction between different chain segments. $\mathrm{Hu}$ et $a .^{5}$ showed that defects in the polymer backbone influence energy transfer directly and indirectly through their affect on conformation. In seeking to determine the origin of this rapid energy transfer, Nguyen et al. ${ }^{6}$ embedded MEHPPV in an ordered matrix in such a way that some, but not all, of the chain segments were oriented and isolated from each other by incorporation into aligned channels within the host. The results indicate that energy transfer along the polymer backbone (orientated sections) is slow relative to interchain energy transfer (in the tangled disordered sections).

In this paper we show that thermalized exciton migration
PACS number(s): 78.55.-m, 36.20.- r, 33.50.-j

along the polymer backbone is very inefficient by directly monitoring the fluorescence time trace and corresponding spectral change of a single, short, purely one-dimensional (rodlike), defect-poor polymer (to eliminate the possible channels for three-dimensional exciton migration). Such limited hopping allows the effective conjugation length of a polymer to be observed in the luminescence properties. Finally, we show that a static disorder induced conjugated length distribution model with limited energy transfer is appropriate to understand the photophysics of an isolated polymer.

\section{EXPERIMENT}

Dioctyloxy PPV (DOO-PPV) has been well studied for its cooperative and stimulated emission properties, ${ }^{7,8}$ evenparity states, ${ }^{9}$ and possible application in ultrafast excitonic switching. ${ }^{10}$ The chemical structure of DOO-PPV is shown in the inset of Fig. 5(b). In this work, polymer close to the persistence length ${ }^{11}$ [molecular weight $\left(M_{n}\right)=8.2 \mathrm{~K}$ Daltons; polydispersity $\left(\delta=M_{w} / M_{n}\right)=2.23 ; \sim 24$ monomers long] was extracted from pristine DOO-PPV by taking advantage of the limited solubility of this symmetrically substituted structure. ${ }^{12}$ This fraction was chosen to ensure that (i) the polymer maintains a one-dimensional rod shape when spin coat in a polystyrene matrix, ${ }^{13}$ (ii) a limited number of excitons should be present ${ }^{14}$ and, (iii) the majority of polymers should be defect free. ${ }^{13}$ The prime motivation is to have an isolated rodlike chain structure for the investigation of the intrinsic properties of the PPV's.

The dispersed single-polymer $\left(10^{-4} \mathrm{M}\right.$ in repeat units) stock solution, after further dilution (factor of $\sim 10^{5}$ ) into a polystyrene matrix (polystyrene: chloroform: toluene $=10 \mathrm{mg}$ : $16 \mathrm{ml}: 4 \mathrm{ml})$, was spin cast $(15 \mathrm{~s}$ at $2000 \mathrm{rps}$ followed by $10 \mathrm{~s}$ at $3000 \mathrm{rps}$ ) onto a fused silica substrate to 
form a thin film with a sample dispersion of between 1 and 20 polymers $/ 100 \mu \mathrm{m}^{2}$. The film was protected from oxygen by sealing in a homemade nitrogen cell. ${ }^{13}$

The single polymers were observed on a sample scanning confocal microscope in epifluorescence mode. ${ }^{13}$ Excitation was provided by a linearly polarized Ar-ion laser $(\lambda$ $=488 \mathrm{~nm}, \mathrm{TEM}_{00}$ mode) which was focused by a $100 \times, 1.3$ NA Nikon fluorescence objective onto the sample plane. The resulting excitation area was $\sim 0.16 \mu \mathrm{m}^{2}$ allowing individual polymers to be clearly resolved. The resulting photoluminescence (PL) was collected by the same objective, and after passing through a long pass filter was recorded by a photoncounting avalanche photodiode (APD) (EG\&G SPCM). After raster scanning the sample under low irradiance, to ensure that single polymers were being observed, the excitation beam was fixed on individual polymers. The intensity of the fluorescence time trace was recorded with $10 \mathrm{~ms}$ time resolution, while the time-averaged spectrum was recorded using a liquid- $\mathrm{N}_{2}$-cooled charge-coupled device (CCD). In contrast to measurements involving large numbers of polymers, the $S / N$ ratio in single molecule experiments is not high enough for the complete spectra to be recorded with $10 \mathrm{~ms}$ time resolution. Thus in order to obtain information on the time evolution of an individual polymer's PL spectrum, it is necessary to split the emission into a few discrete channels. To this end a polarization insensitive beamsplitter $\left(\lambda_{\text {center }}\right.$ $=555 \mathrm{~nm}$ ) was used to split the emitted light ${ }^{15}$ for detection by two calibrated APD's ( $\mathrm{APD}_{\text {blue }}$ detecting from 520 to 555 $\mathrm{nm}$ and $\mathrm{APD}_{\text {red }}$ detecting from 555 to $675 \mathrm{~nm}$ ). By recording the fluorescent transient and then calculating the normalized spectral shift coefficient $S$ defined as

$$
S=\frac{\mathrm{APD}_{\text {red }}-\mathrm{APD}_{\text {blue }}}{\mathrm{APD}_{\text {red }}+\mathrm{APD}_{\text {blue }}}=\frac{1-R}{1+R} ; \quad \text { where } R=\frac{\mathrm{APD}_{\text {blue }}}{\mathrm{APD}_{\text {red }}}
$$

spectral changes were observed with high $S / N$ ratio. (If the PL spectrum does not change with time, $S$ will be constant with time. A gradual change will appear as a slanted line and a sudden spectral jump by a discontinuity.) For example, if an exciton with a slightly blueshifted spectrum (with respect to other excitons) stops emitting, $S$ will suddenly increase. It should be noted that this calculation and conclusion is independent of the calibration of the two collection channels.

\section{RESULTS}

Light emission from electroluminescent organic materials derives from excited molecular states (excitons). ${ }^{16}$ Photoexcitation of a conjugated polymer will generate an electron and hole with opposite spin. In a nondegenerate ground-state polymer, it is expected that this electron and hole become bound, through Coulomb attraction, into a singlet exciton state within few hundred femtoseconds. It is further expected that there is a statistical distribution of segments with various lengths in the polymer due to conformational disorder. ${ }^{25}$ Such disorder induces breaks in the coplanarity between neighbor units in conjugated system. Thermal effect, external force, and angle fluctuating between neighboring units can induce these losses of coplanarity. The length of each seg- ment is determined by the delocalization of a thermalized exciton and is called the effective conjugated length. By comparing the spectral properties of the polymer and that of the corresponding oligomers, the average delocalization length of an exciton is assigned to be about 6-8 repeat units in the poly-( $p$-phenylene vinylene) (PPV's). Since absorption is an instantaneous process, the absorption spectrum reflects the distribution of the delocalization length of the chain segments. However, emission may occur hundreds of picoseconds after excitation. During this time, processes such as the lengthening of the excited segments, energy transfer between neighboring segments or entangling chains, and exciton migration, are all possible pathways for the lowering of emission energy and narrowing of the overall PL spectrum. Timeresolved PL of the polymer thin films on the picosecond time scale reveals a highly disordered density of states. ${ }^{1}$ Within the first $50 \mathrm{ps}$, a spectral redshift of about $30 \mathrm{meV}$ is observed in the polymer films. It is attributed to the inhomogeneously broadened density of states, and the hopping processes from higher-lying localized levels (short conjugated segments) to lower-lying states (longer conjugated segments). As a result, emission occurs mainly from long segments. ${ }^{17}$

In the system investigated here, a polymer close to the persistent length is studied. Diluting the rodlike chain structure simplifies the possible energy relaxation paths by eliminating both interchain and intrachain entanglement. Spectroscopic investigation on an individual polymer removes the effects of ensemble averaging. Our result indicates that each segment has its own characteristic emission spectrum. An abrupt emission intensity jump is usually accompanied by a corresponding spectral change-redshifted or blueshifted. Analyzing the result by a molecular exciton model, it is suggested that the exciton migration along the polymer chain is inefficient. The distribution of chain segments reflects on the statistical result of the time-dependent PL spectrum of the individual polymers.

\section{A. Spectrum and intensity of fluorescence time trace}

Fluorescence time traces were taken for a few hundred polymers. Figure 1 represents a trace in which light is emitted at seven discrete intensities. Changes in intensity are abrupt (as can be seen in the inset) occurring within the 10 -ms temporal resolution of the experiment and may be divided into two categories: recoverable and nonrecoverable intensity drops. The emission sequence in Fig. 1 can be summarized as 6-7-2-5-3-1-2-4-3-2-1-0-1-0. In general, emission is observed at between three and five discrete levels. The lifetime was dependent on the degree to which oxygen was excluded from contact with the sample (from $<1$ s under ambient conditions to $>30$ min in the case of efficient oxygen exclusion). The data are consistent with a view that a polymer is composed of a number of independent excitons. At maximum emission, all are active. Decrease in emission occurs whenever an exciton is either permanently or temporarily disabled from taking part in either absorption or emission (referred to as "dark" or "intermediate" level in Ref. 18). Oxygen, charged species, or conformation twist, can be 


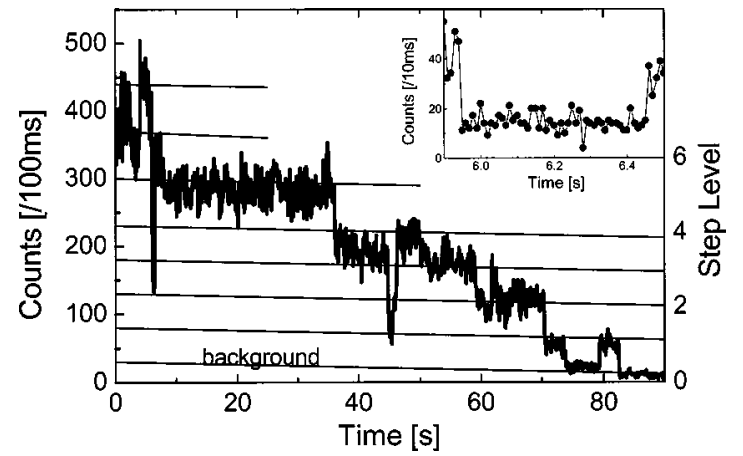

FIG. 1. Typical fluorescent time trace of a single DOO-PPV polymer irradiated by $200 \mathrm{~W} / \mathrm{cm}^{2}$ of linearly polarized $488-\mathrm{nm}$ light. Data are displayed at $100-\mathrm{ms}$ integration time to increase the $S / N$ in the main figure. The inset expands the scale around $6 \mathrm{~s}$ to show that intensity jumps occur abruptly within the 10-ms integration time used in the experiment. The horizontal lines are slightly slanted to compensate to first order for drift (due to the use of an open-loop piezo) and illustrate that emission predominately occurs at seven discrete intensities for this particular polymer.

responsible for the quenching processes. Since the polymer is $\sim 24$ monomers long, four or five excitons would reflect an average conjugation length within the polymer of $\sim$ five or six monomers. ${ }^{19}$

It should be noted that the dark level observed in the present single molecule experiment is likely neither the dipole-forbidden $m \mathrm{Ag}$ state nor the triplet state. In the first case, the optically forbidden $m \mathrm{Ag}$ state, located at $3.3 \mathrm{eV}{ }^{9}$ cannot be directly accessed by the $2.54-\mathrm{eV}$ excitation used in the current experiment. Multiphoton processes appear to be excluded as variation of excitation irradiance from 20 to $1600 \mathrm{~W} / \mathrm{cm}^{2}$ resulted in linear increase in PL intensity and a linear decrease in polymer lifetime as would be expected for a single step process. In the second case, while the lifetime of the triplet state has been seen to be very long at cryogenic temperatures, ${ }^{20}$ at room temperature it declines to the microsecond range as a result of nonradiative decay channels. ${ }^{21}$ Monkman et al. ${ }^{22}$ have measured the lifetime of the triplet state in DOO-PPV, and found it to be $134 \mu$ s when diluted in a benzene solution. While we cannot rule out that this dark state is indeed a triplet state of some sort, the length of time spent at these dark levels (up to tens of seconds) make the triplet state explanation unlikely.

In Fig. 2 the spectrum of two individual polymers are displayed. While the ensemble average of the spectra of many individual polymers resembles that in solution (Fig. 7), each polymer has a unique spectral signature. The singlemolecule spectrum is significantly narrower than the ensemble average. These results suggest that each step in Fig. 1 might exhibit different spectral characteristics. Finally, the lacking of broad featureless emission centered at $650 \mathrm{~nm}$ indicates that excimer emission is absent in the singlemolecule spectrum. ${ }^{23}$

\section{B. Measurement of spectral evolution of the time trace}

The evolution of the single polymer spectrum during the fluorescent time trace provides valuable information on the

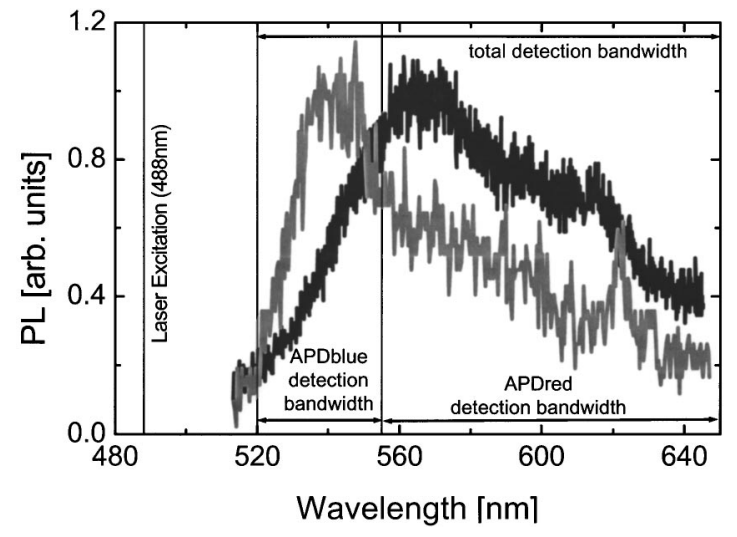

FIG. 2. Room-temperature PL spectra from two single short chain DOO-PPV polymers integrated from initial excitation until completely photobleached. While individual polymers exhibit significantly different behavior, the ensemble average is similar to that taken in solution (cf. Fig. 7). Lines superimposed on the figure indicate the laser excitation frequency and the detection bandwidth of the two APD's used in monitoring the time-dependent spectral changes. The total detection bandwidth was used for the data recorded in Fig. 1. The change in the detection efficiency of the APD's ( $60 \pm 10 \%$ across this bandwidth) was taken into account in the spectral shift calculations.

source of these photons and nature of the excitons emitting them. In addition, by determining the source of these emitted photons, the efficiency of exciton migration can be evaluated. If exciton migration is inefficient along the polymer backbone, the absorption and emission occur at the same conjugated segment. If migration is efficient in the chain, energy will be funneled from absorbing segments to one specific, lower energy site before PL emission. Evidence of energy transfer or its lack can be seen in the behavior of the spectral shift coefficient accompanying an abrupt intensity change. The assumption of a statistical distribution of effective conjugated lengths in the polymer produces the two extreme cases considered in the next paragraphs.

On the one hand, in the case of efficient energy transfer, the emission is taking place at the lowest emission site. The quench itself might take place at any site-including the one responsible for the emission. A few different types of behavior may be observed in this case. (i) The emission intensity may involve totally on-and-off blinking, with no intermediate level being observed. The off state is due to total quenching (if energy transfer still hold to funnel downstream and the site responsible for emission is quenched). (ii) The emission intensity involves blinking with several intermediate levels observed. The partly quenched levels may be either accompanied by no spectral change (if energy is funneled to the lowest emission site, but sites other than the emitting one are quenched), or a blueshifted spectrum at lowering emission intensity (if energy transfer to the lowest site is interrupted by the quenching, and the next-to-lowest one replaces it).

On the other hand, in the case of inefficient energy transfer, the site emitting a photon is the same site that absorbs the photon. In this case, the polymer can be seen as a linkage of 


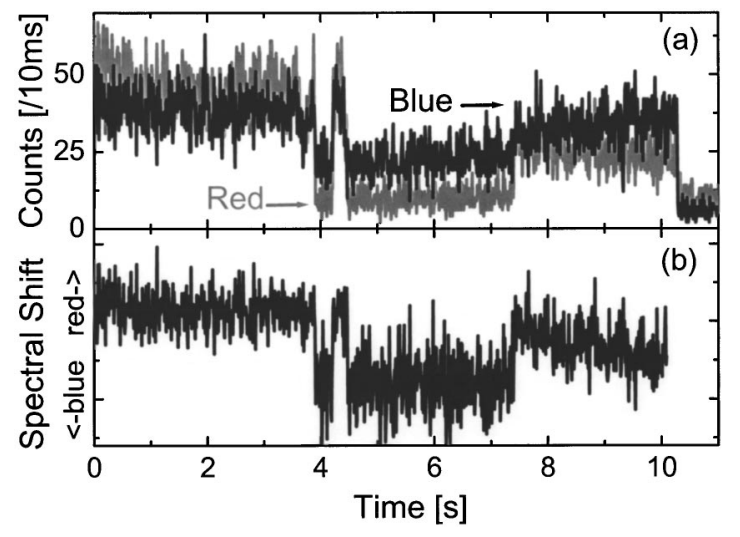

FIG. 3. Fluorescence time trace for a polymer in which intensity drops are accompanied by blue spectral shift. Count rates recorded by the two APD's are shown in (a) and the spectral shift coefficient plotted in (b). Data is taken under constant excitation irradiance $\left(\sim 1400 \mathrm{~W} / \mathrm{cm}^{2}\right)$.

independent chromophores, each is composed of several repeat units, with no cross talking between them. Absorption and emission of a polymer is equal to the sum of the individual chromophores' contribution. Blinking occurs, when one or more chromophores are quenched. The spectral change of the intensity jump depends on the chromophores being quenched. Under the assumption that quench processes have preference neither for the repeat units of the chromophore, nor for the specific site of the chromophore, the statistics of the spectral change should obey the distribution of the energy levels of the chromophores.

Figures 3-5 are typical behaviors of the fluorescence time trace and spectral shift observed in various single polymers. A feature common to all polymers observed is that just as intensity changes are discrete and not gradual, so also is spectral change. These results indicate each exciton has its distinctive spectral characteristics, which remain constant on the hundred-millisecond time scale of this experiment.

Looking first at Fig. 3, intensity jumps occur simultaneously in the raw data of both channels (a). The relative

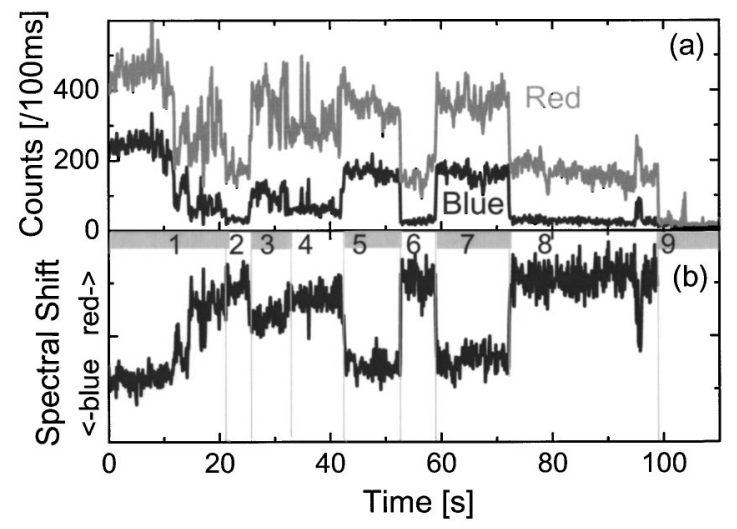

FIG. 4. Fluorescence time trace for a polymer in which intensity drops are accompanied by a red spectral shift. Count rates recorded by the two APD's are shown in (a) and the spectral shift coefficient plotted in (b). Data are taken under constant excitation irradiance $\left(\sim 1400 \mathrm{~W} / \mathrm{cm}^{2}\right)$.

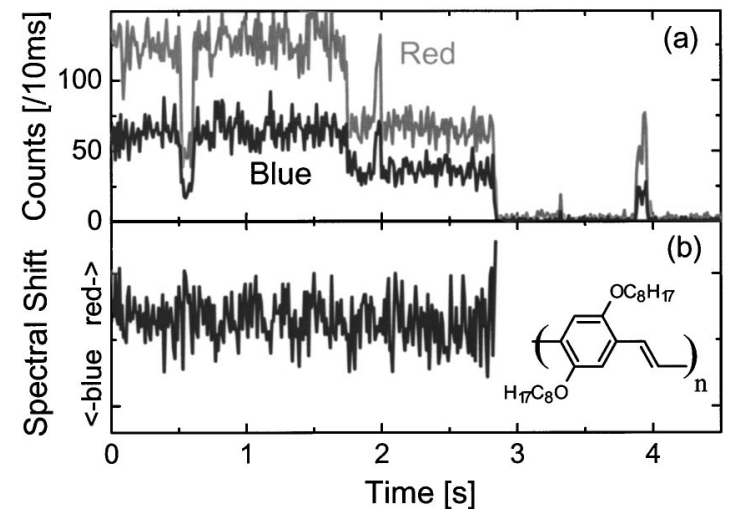

FIG. 5. Fluorescent time trace for a polymer exhibiting in which there is no spectral shift accompanying intensity changes. Count rates recorded by the two APD's are shown in (a) and the spectral shift coefficient plotted in (b). Data are taken under constant excitation irradiance $\left(\sim 1400 \mathrm{~W} / \mathrm{cm}^{2}\right)$. The chemical structure of DOOPPV is shown in the inset.

magnitude of the jumps, however, differs-indicating a change in the spectral composition of the emission. This is seen more clearly in Fig. 3(b), where the normalized spectral shift coefficient is plotted. It can be observed that every intensity jump is accompanied by a corresponding spectral jump. In addition, lower intensity emission is blueshifted relative to the higher emission level.

Figure 4 shows another typical trace. As with the polymer shown in Fig. 3, intensity jumps are accompanied by a corresponding spectral jump. However, in contrast to the previous polymer (Fig. 3) where intensity drops result in a spectral shift to the blue, intensity drops in this polymer are accompanied by a spectral shift to the red. In Fig. 4 the emission is divided into nine regions. In the first region there are quite a few intensity and spectral jumps occurring on a time scale of a few seconds. By the ninth region, emission has stopped. After the first $21 \mathrm{~s}$ the emission becomes more stable and thus our discussion will focus on regions $2-8$. In regions 2 (21-25 s), 6 (53-59 s), and 8 (72-99 s), both total intensity and spectral shift are almost the same. This implies that the source is either the same segment or the same group of segments. However, in regions 3 (25-32 s) and 5 (42-53 s), despite the fact the total intensity (calibrated sum of counts measured by $\mathrm{APD}_{\text {blue }}+\mathrm{APD}_{\text {red }}$ ) is similar, the spectral shift coefficient differs greatly in these two regions. This suggests that they belong to a different source of the emission. One explanation is that one exciton emits light from the beginning until it is quenched at the end of region 8 ( $99 \mathrm{~s}$ ). In the regions 3,5 , and 7 , additional excitons contribute to additional intensity. The spectral shift indicates the same combination of excitons emits in regions 5 and 7 while a different combination emits in region 3.

Over 46 individual polymers were considered in this study. In 11 cases, intensity drops were accompanied by a blue spectral shift as in Fig. 3. In an additional 15 polymers intensity drops were accompanied by red spectral shift. Six polymers exhibited a combination of blue and red spectral shifts. In five polymers, intensity jumps not accompanied by a spectral shift were observed. The transient time trace of 


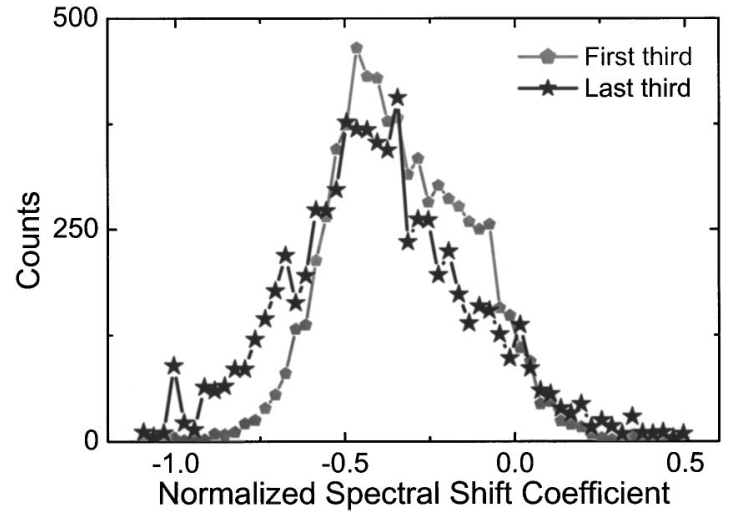

FIG. 6. Histogram of calibrated spectral shift coefficients for over 30 polymers comparing the observed spectral shift during the first third and last third of each polymer's lifetime. Every point in the spectral jump diagram of these molecules was taken into account.

one such representative polymer is shown in Fig. 5. Although intensity jumps are clearly seen at $0.5-\sim 0.6 \mathrm{~s}, 1.75-\sim 2 \mathrm{~s}$, there was no change in the spectral shift coefficient accompanying any of these intensity jumps. Two possible explanations exist. The first is that there are two perfectly identical independent segments. The second is that energy is transferred efficiently between two segments with intensity jumps occurring whenever the higher energy site enters or leaves a dark state. While individual experimental results alone do not allow us to distinguish between these two explanations, the fact that this type of behavior is so rarely observed leads us to prefer the first explanation.

Another evidence to support the inefficient energy transfer is the evolution of the spectral shift histogram with time. Since, before the experiments, the polymer can stay a long time in a dark environment without degrading, it is reasonable to assume the rate of bleaching to be proportional to the time spent in the excited states. When energy transfer from higher to lower energy excitons is efficient, one expects lower energy sites to bleach first, resulting in a progressively blueshifted spectrum with time. Figure 6 compares the probability of a given spectral shift occurring during the first recorded third and final third of each of individual polymer's lifetime. Only a slight blueshift with time is observed in the histogram. We conclude that there is only very limited energy transfer along the polymer backbone.

From these experimental results we conclude that, unlike (long-chain) MEH-PPV both in solution ${ }^{17}$ and polymer matrix, ${ }^{4}$ energy transfer in short rodlike DOO-PPV polymer is remarkably inefficient. Exciton migration along the polymer backbone seems not to have occurred before luminescence. A result of this is that the distribution of effective conjugation length can be observed during the lifetime of a polymer. In the following discussion, we will further apply a molecular exciton model to aid in the interpretation of these results.

\section{DISCUSSION}

In analyzing the data, we have employed a molecular exciton model in which the polymer is considered to be com- posed of different conjugated segments whose appearance is governed by a distribution function. This model has been adequately applied to explain the chain-length dependence of the absorption spectrum and third-order nonlinear susceptibilities of the well-defined linear polyene oligomers, ${ }^{24}$ as well as the steady-state absorption and PL spectrum of PPV derivatives in solution. ${ }^{17}$ When the polymer is embedded in a solid solution, this disorder induced conjugated length distribution model should be applicable. This phenomenological treatment is suitable for understanding long time ( $\gg 100 \mathrm{ps}$ ) photophysics processes - the time regime in which this single-molecule experiment was performed. In brief (a fuller description is available in Ref. 17), the absorption coefficient $(\alpha)$ and the intensity $(I)$ of the PL spectrum are given by

$$
\alpha(\omega)=\sum_{N} D[N] \alpha_{N}(\omega)
$$

and

$$
I(\omega)=\sum_{N} \rho[N] D[N] I_{N}(\omega)
$$

respectively. $\alpha_{N}(\omega)$ and $l_{N}(\omega)$ represent the absorption and PL spectrum of a single $N$-unit conjugated segment (henceforth referred to as oligomer). $D[N]$ denotes the probability of finding an oligomer having $N$-units oligomer in the chain. $D[N]$ is calculated based on a conformational disorder model, ${ }^{25}$ as this is considered to be the main cause of finite conjugation length in a conjugated polymer. ${ }^{17}$ This model proposes a Gaussian distribution for $D[N]$, i.e.,

$$
D[N]=\frac{1}{\sqrt{\pi B_{a v}}} \exp \left[-\frac{\left(N-N_{0}\right)^{2}}{B_{a v}}\right] .
$$

The center $\left(N_{0}\right)$ and width $\left(B_{a v}\right)^{1 / 2}$ are adjustable parameters to be determined by fitting both the absorption and PL spectrum.

Finally,

$$
\rho[N]=\frac{\exp \left(-E_{N_{b}} / k_{B} T\right)}{\sum_{N} \exp \left(-E_{N_{b}} / k_{B} T\right)}
$$

represents the relative weighting of each occurring oligomer's contribution to the PL spectrum under the assumption that thermal equilibrium within all electronic states has been reached prior to emission. At room temperature, this last assumption is equivalent to assuming that rapid energy transfer occurs within the polymer. The inset in Fig. 8 shows the absorption and PL spectra of different oligomers used in the model. The Huang-Rhys parameter, as estimated by the ratio of secondary vibronic peak (1-0 transition) to the first peak (0-0 transition) in the fluorescence spectrum, indicates the strength of electron-phonon coupling. In the present model the long chain oligomer has weaker electron-phonon coupling, consistent with theoretical calculations and previous experimental work. ${ }^{27}$ This model has been applied to $\mathrm{MEH}-$ PPV (Ref. 17) and DOO-PPV (Ref. 26) in chloroform solvent. 


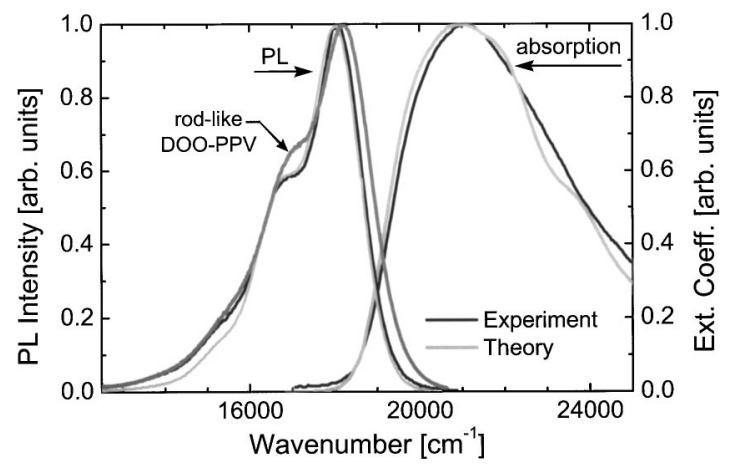

FIG. 7. The calculated and measured ensemble absorption and PL spectra of DOO-PPV in room-temperature chloroform. Experimental results are shown by dark lines and theoretical results are shown by light gray lines. The calculation is based on rapid energytransfer model discussed in the text. The measured PL for long chain is shown in black and the measured PL for the short chain sample is presented in dark gray.

The experimental absorption and PL spectra for long (molecular weight $\gg 100000$ ) and short chain rodlike DOOPPV, along with theoretical fitting using above model is shown in Fig. 7. There is excellent agreement between experiment and theory for the long chain polymer with the theoretical calculation laying almost on top the experimental curves. This supports the argument that energy transfer in such tangled polymers is rapid (i.e., Eq. (2d) is valid). Considering the PL spectrum of the short chain polymer, two observations can be made: it is slightly blue shifted from the long chain results as well as being significantly wider (2400 $\mathrm{cm}^{-1}$ vs $2150 \mathrm{~cm}^{-1}$, respectively). ${ }^{12}$ The additional width indicates that there are a greater variety of oligomers emitting in the rodlike polymer than in the longer polymer. While it may be possible to adjust model parameters to take into account the slight blueshift, it is not possible to explain the additional spectral width using the above model with the assumption of rapid energy thermalization [Eq. (2d)].

The application of the model to explain the histogram obtained in the double port experiment can be broken into three steps. First, from the theoretical absorption $\left[\alpha_{N}(\omega)\right]$ and PL spectrum $\left[I_{N}(\omega)\right]$ of conjugated segments composed of $N=3$ to $N=9$ monomer units shown in the inset of Fig. 8, the expected ratio of signals on the two APD's $\left[R_{\text {theory }}(N)\right]$ under experimental conditions was calculated for each oligomer taking into account the wavelength dependent efficiencies of the detectors and all elements in the optical train. From this the theoretical spectral shift coefficient for an oligomer of length $N$ was obtained using Eq. (1).

Second, the probability of the appearance of each oligomer $(D[N])$ was calculated using the values for these parameters published by Chang et al. ${ }^{17} \quad\left[N_{0}=5\right.$ and $\left.\left(B_{a v}\right)^{1 / 2}=1.8\right]$.

The third and final step involves determining the probability of a given spectral shift being observed experimentally in the PL spectrum. This involves determining the relative weighting of each occurring oligomer's contribution to the PL spectrum $(\rho[N])$. This weighting factor is strongly dependent on the model of energy transfer used. Two limiting

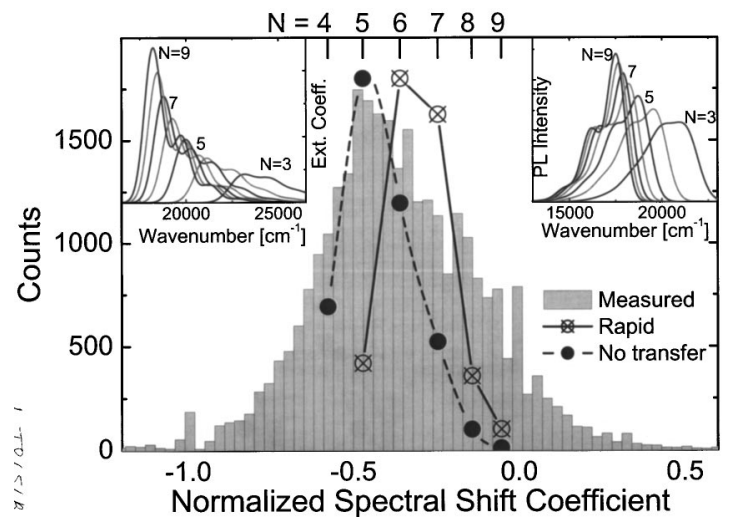

FIG. 8. Histogram of calibrated spectral shift coefficients for over 30 polymers. Every point in the spectral jump diagram of these molecules was taken into account. Superimposed on the diagram is theoretical probability for a given spectral shift occurring in the limits of no energy transfer between oligomers (solid circles) and rapid energy transfer between oligomers (crossed open circles). The number on the upper horizontal axis indicates the size of the oligomer. The insets present the calculated relative extinction coefficients (left) and PL spectra (right) for oligomers with $N=3-9$. Odd numbered oligomers are shown in black while even numbered oligomers are shown in gray. PL spectra profiles are normalized to unity area.

cases of rapid energy transfer and absolutely no energy transfer are considered. In the absence of exciton migration (the oligomer that absorbs a photon emits a photon) the relative weighting of an occurring oligomer's contribution to PL is simply its extinction coefficient at the excitation wavelength (in our case, $488 \mathrm{~nm}$ ) relative to the other oligomers. (Note that while the distribution function indicates $6 \%$ of the oligomers have $N=2$; however, since $N=2$ oligomers do not absorb at $488 \mathrm{~nm}$, they make no contribution.) This can be expressed mathematically as $\rho[N]=\alpha_{N}(488 \mathrm{~nm})$.

In the case of efficient migration to the lowest energy oligomer, the relative weighting of an oligomer's contribution is proportional to its probability of being the lowest energy (longest) oligomer in a polymer $\left(p_{\text {longest }}[N]\right)$. This was calculated by theoretically creating 400 polymers (each $\sim 24$ repeat units) by progressively adding oligomers whose length was chosen by random sampling from the distribution function $(D[N])$. A point was scored by the longest oligomer in each polymer. (For example, if sampling gives the string of numbers $7,5,5,4,5,4,4,7 \cdots$ the constructed polymer would be composed of oligomers: 7-5-5-4-5 (length=26) and the longest oligomer $(N=7)$ would score one point). This can be expressed mathematically as $\rho[N]$ $=p_{\text {longest }}[N] / D[N]$.

In Fig. 8 the experimentally derived spectral shift histogram along with the results of this calculation for the two limiting cases of no energy transfer and efficient energy transfer between conjugated segments (denoted as closed and open crossed circles, respectively) normalized to experiment. Comparing the calculated and measured histograms, one is struck by the good agreement between experiment and the no energy-transfer calculation. The most probable value of the spectral shift coefficient is nearly identical for both the ex- 
perimentally derived and that calculated theoretically. In contrast, the peak of the efficient energy transfer calculation is considerably shifted to the red relative to the experimental results. While the experimental histogram is slightly wider than that calculated, this is to be expected as we have used the value for $B_{a v}$ characteristic for the polymer in solution. It is reasonable to expect that in a solid solution the distribution will have a larger half width as each conjugated segment will experience a larger environmental inhomogeneity. Including this in the model would only improve the correspondence with the no energy-transfer calculation and further reduce the correspondence with the efficient energy-transfer calculation. This inefficient energy transfer in a rodlike polymer allows the effective conjugation length of an individual polymer to be observed not only in the absorption but also in the fluctuation of the single polymer fluorescence.

At this point we would like to speculate briefly on the physical basis for this inefficiency of energy transfer along the polymer backbone (in a single rodlike polymer, threedimensional energy transfer is not possible). First, Förster energy transfer will be relatively inefficient as there is, in general, very little spectral overlap between the absorption and emission of adjacent segments along the polymer. For example, in the "model" polymer discussed above (7-5-5-45), while the $N=5 \rightarrow N=7$ transition exhibits significant spectral overlap, and some overlap also exists for the $N=4$ $\rightarrow N=5$ transition, for the remaining transition $(N=5 \rightarrow N$ $=4$ which represents an increase in energy and $N=5 \rightarrow N$ $=5$ ), there is no spectral overlap. Thus, for example, if the $N=5$ oligomer on the right end is excited, the energy cannot transfer efficiently to $N=7$ oligomer at the other end. Second, energy transfer is strongly dependent on the separation $\left(1 / r^{6}\right)$ between excitons. In a rodlike polymer the separation between the centers of adjacent conjugated segments is $\sim 4$ $\mathrm{nm}$ - too large for efficient energy transfer. When the intermediate segment's exciton enters a dark state, the ability of neighboring excitons to "hop" between segments becomes even more difficult. A final factor mitigating against the migration of thermalized excitons is the short excited state lifetime ( $\sim 500 \mathrm{ps}$, an order of magnitude shorter than the $\sim 5 \mathrm{~ns}$ typical of dyes). The short excited state lifetime implies less energy transfer efficiency, assuming the same Förster energy transfer rate. Note that the above arguments are only qualitative. Because the distance between excitons is similar to exciton size in conjugated polymers, a generalized Förster theory ${ }^{28}$ might be required to perform quantitative energytransfer calculation. Two recent papers, one by Beljonne et $a l .{ }^{29}$ using a specially designed molecule and ultrafast spectroscopy and the other by Nguyen et al. ${ }^{6}$ have also concluded that intrachain energy transfer is inefficient in rodlike polymers.

The issue of defect-induced exciton quenching (both permanent and temporary) is an important one in polymer physics. For example, Rothberg et al. ${ }^{2}$ reported that the quenching radius of MEH-PPV thin film by the photo-oxidation induced $\mathrm{C}=\mathrm{O}$ bond is $\sim 400$ monomer units. Since in films excitons can move in three dimensions, this implies an exciton will be influenced by defects within a 5-nm radius. The lack of exciton migration in a rodlike polymer might then greatly reduce the effect of a defect to those conjugated segments nearest to the defect-a view that is supported by the experimental data. Considering the polymer represented in Fig. 3, its spectrum is consistently blueshifted at the lower intensity and redshifted when emission recovers to the original emission intensity. This type of behavior (or the opposite in which the emission at the lower intensity was redshifted relative to "full" intensity, Fig. 4) was observed for over $75 \%$ of the polymers observed. It can be explained in the absence of energy transfer if the conjugated segment closest to a quencher molecule (i.e., $\mathrm{O}_{2}$ ) experiences its effect most intensely while those segments farthest away are the least affected. For example, taking our sample polymer, 7-5-5-4-5. If a quencher molecule is near the right end, then the $N=5$ segment will experience its effect most directly and the emission will be redshifted at lower intensity. On the other hand, if the quencher lies near the left end, then the $N=7$ segment will experience the effect most intensely and the emission will be blueshifted at lower intensity.

The lack of exciton migration along the polymer backbone also indicates why single long chain MEH-PPV properties depend so strongly on processing conditions. ${ }^{4}$ As the polymer's length is much longer than its persistent length, it tends to fold into defect-coil and defect-cylinder structures, ${ }^{5}$ in which many paths exist for site-to-site energy transfer. For example, in a coiled structure, the distance between the center of two conjugated segments in different sections of the polymer is much less than between two adjacent segments. In addition, there is a high probability of segments having very different conjugation lengths being adjacent, resulting in a high value for the spectral overlap integral (efficient Förster energy transfer). These effects would facilitate fast three-dimensional exciton migration relative to that along the polymer backbone. As a result, energy transfer will be highly dependent on the exact three-dimensional conformation taken by the polymer and thus a function of processing conditions.

\section{CONCLUSIONS}

In conclusion, the exciton migration mechanism in, and photophysics of, a rodlike polymer is substantially different from a long chain, folded polymer. Migration of thermalized excitons is inefficient along the polymer backbone. Thus the effective conjugation length of the polymer can be observed by the single-molecule fluorescence. It should be possible to reduce defect quenching by using isolated polymers having a straight backbone. This would allow devices to be built with considerably longer lifetimes.

\section{ACKNOWLEDGMENTS}

We acknowledge discussions with R. L. Chang and K. K. Liang. This research was supported by the National Science Council, ROC, and MOE Program for Promoting Academic Excellence of Universities under Grant No. 91-E-FA04-2-4A. 
${ }^{1}$ R. Kersting, U. Lemmer, R. F. Mahrt, K. Leo, H. Kurz, H. Bassler, and E. O. Gobel, Phys. Rev. Lett. 70, 3820 (1993).

${ }^{2}$ L. J. Rothberg, M. Yan, F. Papadimitrakopoulos, M. E. Galvin, E. W. Kwock, and T. M. Miller, Synth. Met. 80, 41 (1996).

${ }^{3}$ J. Yu, D. Hu, and P. F. Barbara, Science 289, 1327 (2000).

${ }^{4}$ T. Huser, M. Yan, and L. J. Rothberg, Proc. Natl. Acad. Sci. U.S.A. 97, 11187 (2000).

${ }^{5}$ D. Hu, J. Yu, K. Wong, B. Bagchi, P. J. Rossky, and P. F. Barbara, Nature (London) 405, 1030 (2000).

${ }^{6}$ T. Q. Nguyen, J. Wu, S. H. Tolbert, and B. J. Schwartz, Adv. Mater. 13, 609 (2001).

${ }^{7}$ S. V. Frolov, W. Gellermann, M. Ozaki, K. Yoshino, and Z. V. Vardeny, Phys. Rev. Lett. 78, 729 (1997).

${ }^{8}$ S. V. Frolov, Z. V. Vardeny, and K. Yoshino, Phys. Rev. B 57, 9141 (1998).

${ }^{9}$ S. V. Frolov, Z. Bao, M. Wohlgenannt, and Z. V. Vardeny, Phys. Rev. Lett. 85, 2196 (2000).

${ }^{10}$ S. V. Frolov, P. A. Lane, W. Gellermann, and Z. V. Vardeny, Phys. Rev. Lett. 78, 4285 (1997).

${ }^{11}$ C. L. Gettinger, A. J. Heeger, J. M. Drake, and D. J. Pine, J. Chem. Phys. 101, 1673 (1994).

${ }^{12}$ J. H. Hsu, M. Hayashi, S. H. Lin, W. S. Fann, L. J. Rothberg, G. Y. Perng, and S. A. Chen, J. Phys. Chem. B 106, 8582 (2002).

${ }^{13}$ J. D. White, J. H. Hsu, W. S. Fann, Shu-Chun Yang, G. Y. Pern, and S. A. Chen, Chem. Phys. Lett. 338, 263 (2001).

${ }^{14}$ M. Yan, L. J. Rothberg, F. Papadimitrakopoulos, M. E. Galvin, and T. M. Miller, Phys. Rev. Lett. 73, 744 (1994).

${ }^{15}$ T. Ha, Th. Enderle, D. S. Chemla, P. R. Selvin, and S. Weiss, Phys. Rev. Lett. 77, 3979 (1996).

${ }^{16}$ D. D. C. Bradley, J. Phys. D 20, 1389 (1987).

${ }^{17}$ R. Chang, J. H. Hsu, W. S. Fann, K. K. Liang, C. H. Chang, M.
Hayashi, J. Yu, S. H. Lin, E. C. Chang, K. R. Chuang, and S. A. Chen, Chem. Phys. Lett. 317, 142 (2000).

${ }^{18}$ D. A. Vanden Bout, W. T. Yip, D. Hu, D.-K. Fu, T. M. Swager, and P. F. Barbara, Science 277, 1074 (1997).

${ }^{19}$ J. D. White, J. H. Hsu, Shu-Chun Yang, W. S. Fann, G. Y. Pern, and S. A. Chen, J. Chem. Phys. 114, 3848 (2001).

${ }^{20}$ D. Comoretto, I. Moggio, C. Cuniberti, G. Dellepiane, M. E. Giardini, and A. Borghesi, Phys. Rev. B 56, 10264 (1997).

${ }^{21}$ C. Rothe, S. Hintschich, A. P. Monkman, M. Svensson, and M. R. Anderson, J. Chem. Phys. 116, 10503 (2002).

${ }^{22}$ A. P. Monkman, H. D. Burrows, L. J. Hartwell, L. E. Horsburgh, I. Hamblett, and S. Navaratnam, Phys. Rev. Lett. 86, 1358 (2001).

${ }^{23}$ J. H. Hsu, W. S. Fann, P. H. Tsao, K. R. Chuang, and S. A. Chen, J. Phys. Chem. A 103, 2375 (1999).

${ }^{24}$ B. E. Kohler and I. D. W. Samuel, J. Chem. Phys. 103, 6248 (1995); B. E. Kohler, J. C. Woehl, J. Chem. Phys. 103, 6253 (1995).

${ }^{25}$ G. Rossi, R. R. Chance, and R. Silbey, J. Chem. Phys. 90, 7594 (1989).

${ }^{26}$ R. Chang, J. H. Hsu, W. S. Fann, J. Yu, S. H. Lin, Y. Z. Lee, and S. A. Chen, Chem. Phys. Lett. 317, 153 (2000).

${ }^{27}$ J. Cornil, D. Beljonne, Z. Shuai, T. W. Hagler, I. Campbell, D. D. C. Bradely, J. L. Brédas, C. W. Spangler, and K. Müllen, Chem. Phys. Lett. 247, 425 (1995); J. Cornil, D. Beljonne, C. M. Heller, I. H. Campbell, B. K. Laurich, D. L. Smith, D. D. C. Bradely, K. Müllen, and J. L. Brédas, ibid. 278, 139 (1997).

${ }^{28}$ G. D. Scholes, X. J. Jordanides, and G. R. Fleming, J. Phys. Chem. B 105, 1640 (2001).

${ }^{29}$ D. Beljonne, G. Pourtois, C. Silva, E. Hennebicq, L. M. Herz, R. H. Friend, G. D. Scholes, S. Steayesh, K. Mullen, and J. L. Bredas, Proc. Natl. Acad. Sci. U.S.A. 99, 10982 (2002). 